\title{
Single carbon and glass fibre properties characterised using large data sets obtained through automated single fibre tensile testing
}

\author{
Francisco Mesquita $^{1 *}$, Steve Bucknell ${ }^{2}$, Yann Leray ${ }^{2}$, Stepan V. Lomov ${ }^{1}$, Yentl Swolfs ${ }^{1}$ \\ ${ }^{1}$ Department of Materials Engineering, KU Leuven, Kasteelpark Arenberg 44 box 2450, 3001 Leuven, \\ Belgium \\ ${ }^{2}$ Dia-Stron Ltd., 9 Focus Way, Andover SP10 5NY, United Kingdom \\ *Corresponding author: francisco.mesquita@kuleuven.be, +3216373686
}

\begin{abstract}
Large data sets of fibre mechanical properties were acquired using automated single fibre tensile testing equipment to improve the reliability of the measurements on T700S, 34-700, T300, HS40 and HYBON 2026 fibres. Although with the same nominal strength and stiffness, the T700S and 34-700 showed different Weibull modulus. A high number (over 200) of the tests is necessary for accurate determination of the fibre strength Weibull parameters; samplings of $\sim 50$ tests can lead to a Weibull modulus deviation of \pm 1 . Carbon fibre stiffening rate was shown to depend on the fibre type, contrary to previous reported observations. The stiffness of E-glass fibre, however, decreased with the applied strain. The non-linearity of the fibre stress-strain diagrams implies that the necessary compliance calibration should be done iteratively.
\end{abstract}

Keywords: Fibres, Strength, Elasticity, Mechanical testing

\section{Introduction}

The manufacturing process of fibres introduces defects in their microstructure. As carbon and glass fibres are considered to be brittle materials, their tensile strength is strongly influenced by the presence of these defects $[1,2]$. The tensile strength of these fibres therefore cannot be fully characterised by an average value but rather should be characterised by a probability distribution. The Weibull distribution is the most commonly used to characterise the strength of carbon and glass fibres [2-5] and appears to be a good approximation when weakest-link behaviour is present [6,7]. The two-parameter distribution is characterised by the Weibull scale parameter and Weibull modulus. The scale parameter is related to the position of the distribution peak while the modulus governs the width of the distribution. The Weibull 
scale parameter depends on the fibre length due to higher probability of finding a critical defect in a longer fibre. The Weibull modulus has been postulated to describe well the strength variation of strength across a fibre bundle but not along a fibre bundle. Watson and Smith [7] proposed a length scaling parameter that describes a lower strength variation along the fibre length than across a fibre bundle. This length scaling effect has been shown to be more significant for some carbon fibres [8-12]. A strong length scaling effect was also observed for Kevlar fibres [13]. A three-parameter Weibull distribution can also be used and was recently proposed by Islam et al. [14]. They have justified this approach with the absence of T700S carbon fibres weaker than $2 \mathrm{GPa}$ in their datasets. The most common method to obtain the Weibull distribution for fibre strength is the single fibre tensile test [15]. Using this test, the individual fibre stiffness and strength can be obtained directly from the test results. This is a time-consuming method though, as each fibre must be picked from a bundle and its diameter measured before the test. Other methods such as the single fibre fragmentation test or dry bundle test can reduce the testing time $[16,17]$. These methods require a data reduction scheme, introducing assumptions in the determination of fibre strength. There is therefore a balance between the time consumption and the accuracy of the test in acquiring a large data set.

Obtaining large data sets of fibre strength is important to accurately characterise the fibre strength through a Weibull distribution. However, there is no universal number of tests that suits all fibre types as the number of tests necessary to accurately characterise the fibre strength depends on the Weibull modulus [18]. Many authors have attempted the characterisation of the fibre strength through 20-50 tests $[6,12,14,18-32]$; in contrast, Berger and Jeulin [26] showed through a modelling study that assuming a Weibull modulus equal to 4 and performing 30 tests would produce a deviation of \pm 2 in the estimation of the Weibull modulus. If the number of tests would be increased to 180 , the deviation would reduce to \pm 1 . More recently, Swolfs et al. [33] evaluated the influence of the number of tests on the estimation of the Weibull parameters through modelling. They concluded that performing 25-100 tests would lead to significant errors.

With the introduction of automated testing machines by Dia-Stron Ltd. [34] or Textechno [35], the time consumption in single fibre testing is strongly reduced. In this work, the equipment from Dia-Stron Ltd. is used to characterise the tensile strength of four different carbon fibres and one glass fibre type. Large data 
sets are used to characterise the fibre diameter, stiffness and strength, increasing the reliability of the measurement distributions. The limits for reduction of the statistical sampling size without loss of precision of the Weibull parameters are determined by analysis of smaller data sub-sets. The work aims to (1) help the modelling community to fine tune their input parameters and (2) aid the measurement of other properties that require the fibre diameter and/or stiffness.

\section{Materials and methods}

\subsection{Materials}

\subsubsection{Single fibres}

Four different types of PAN-based carbon fibres and one type of E-glass fibre were analysed in this article. The nominal properties of each fibre type, specified by the manufacturer, and the number of fibres tested are shown in Table 1.

The T700S and 34-700 fibres have similar stiffness and tensile strength but are produced by different manufacturers. The goal is to compare the properties of these two fibres and determine if there are differences in their Weibull distributions that may lead to different composite behaviour. The T300 fibre has the same stiffness as the T700S but lower tensile strength. It is expected that the T300 fibre has a lower Weibull scale parameter but it is not straightforward to predict the variation in Weibull modulus. The HS40 fibre is almost twice as stiff as the other fibres but is slightly weaker than the T700S and 34700 fibres. The Weibull scale parameter is therefore also expected to be similar for HS40, T700S and 34700 fibres; HS40 should have a lower failure strain. The HYBON 2026 glass fibre has 1/3 of the tensile modulus of T700S carbon fibre but more than $1 / 2$ of its tensile strength. The failure strain of this fibre should therefore be higher than for the carbon fibres.

\subsubsection{Composite specimens}

In addition to single fibres tests, composite specimens were used to indirectly measure the fibre tensile modulus in a composite and compare it with the modulus measured on single fibres. All the composites were manufactured through the use of ThinPreg ${ }^{\mathrm{TM}}$ 736LT prepregs sourced from North Thin Ply Technology. The prepregs included the 736LT epoxy resin in combination with either HS40 or HYBON 
2026 fibres. The nominal thickness of the HS40/736LT prepregs was $50.8 \mu \mathrm{m}$ and it was $34.5 \mu \mathrm{m}$ for the HYBON 2026/736LT prepregs. The specimens were produced through autoclave curing following the guidelines provided by the manufacturer. A temperature of $70^{\circ} \mathrm{C}$ was maintained for 1 hour before reaching the curing temperature of $120^{\circ} \mathrm{C}$ that was maintained for $45 \mathrm{~min}$. The temperature was increased and decreased at a rate of $2^{\circ} \mathrm{C} / \mathrm{min}$. The overpressure in the autoclave was 7 bar and a vacuum of $-0.7 \mathrm{bar}$ was applied throughout the curing cycle.

The composite specimens were unidirectional to facilitate the data reduction and determination of the fibre tensile modulus. Ten specimens of each type were manufactured. The thickness of the HS40/736LT and HYBON2026/736LT specimens was $1.02 \pm 0.02 \mathrm{~mm}$ and $1.11 \pm 0.03 \mathrm{~mm}$. A total of 20 plies were used for the HS40/736LT specimens and 32 plies were used for the HYBON 2026/736LT specimens. A diamond-coated rotating blade was used to cut the manufactured plate into $15 \times 250 \mathrm{~mm}$ specimens.

\subsection{Methods}

\subsubsection{Automated single fibre testing}

The single fibre tests were performed with the LEX/LDS automated testing equipment developed by DiaStron Ltd [34]. Each fibre is extracted from the bundle by hand with the assistance of a vacuum pen and then mounted onto two plastic tabs, where each tab holds one of the fibre ends. The authors tried to separate the fibre roving into smaller bundles, thus preventing to some extent the breakage of the weaker fibres. Among the carbon fibres, the T700 and T300 carbon fibres were noticeably more difficult to extract due to the presence of a sizing that would stick the fibres together. The HYBON 2026 glass fibres, due to their larger diameter, were also easier to extract without breaking the fibre. The tabs are placed in a cassette with slots for multiple fibres (see Figure 1a). The cassette helps in obtaining an accurate gauge length by providing the slots for the plastic tabs with a pre-defined distance between them $(12.24 \pm 0.10$ $\mathrm{mm}$ in this work). The gauge length was chosen from a set of pre-defined lengths available for the equipment. The minimum gauge length possible was $4 \mathrm{~mm}$ but it was not chosen because: (1) a shorter gauge length would be required to perform the compliance calibration and (2) the fibre misalignment issues become more predominant as the gauge length decreases. For shorter gauge lengths, one would have to perform other type of tests such as single fibre fragmentation or loop tests [15] but these tests do 
not yield the information on the fibre properties directly. Each tab has a V-shaped slit that helps aligning the fibre. A droplet of an ultraviolet (UV) curing adhesive is then placed over each tab to fix the fibre on the tab. The adhesive is cured by illuminating it with a UV lantern for 15 seconds (see Figure 1b). The entire fibre mounting procedure takes 1-2 minutes per fibre. For more details on the procedure, the reader in referred to the work of Islam et al. [14].

When all the slots in the cassette have been filled, the cassette is mounted on the testing equipment. From this moment on, the procedure is automated. Each fibre is automatically picked up from the cassette and placed on the LEX 820 tensile testing machine. The equipment is a combination of an LDS0200 laser diffraction system for the diameter measurement and a tensile tester (see Figure 1c). It is worth mentioning that the LDS0200 system was referenced against calibration carbon fibres tested at METAS. The diameter of each fibre is measured at one axial position on the fibre and at one angular position of the laser. For each fibre, the reported value of the fibre diameter is taken in the same axial and radial position. The tensile tests were carried out with a cross-head displacement rate of $0.6 \mathrm{~mm} / \mathrm{min}$.

\subsubsection{Determination of fibre properties}

The automated single fibre testing machine outputs the fibre diameter and the force-displacement curve for each fibre. Using the fibre diameter, $d_{f}$, and the force applied to the fibre, $F$, the stress, $\sigma_{f}$ can be immediately determined through equation 1 :

$\sigma_{f}=\frac{4 \cdot F}{\pi \cdot d_{f}^{2}}$

The goal is to determine the stress-strain diagram curve which gives access to the elastic modulus and strength. The strain cannot be obtained directly from the displacement and gauge length due to the compliance of the machine. The machine compliance, defined as the inverse of its stiffness, needs to be determined from the test data before the fibre tensile modulus can be calculated. The methodology to determine the machine compliance is described in ASTM C1557-14 [36]. It encompasses the testing of fibres at different ratios of gauge lengths to the fibre diameter (see Figure 2a). This was done by using nominal gauge lengths of 4 and $20 \mathrm{~mm}$ in addition to the gauge length of $12 \mathrm{~mm}$ used to obtain the large 
data sets. The exact gauge length was determined for each fibre by extrapolating the load-displacement curve and finding the intersection with the horizontal axis.

Prior to the tensile test, the fibres are made straight by applying a force $F_{i}=0.01 \mathrm{~N}$ so that their diameter is properly measured. The first point in the stress-strain diagram therefore corresponds to an initial strain, $\varepsilon_{i}$, that can be estimated through equation 2 :

$\varepsilon_{i}=\frac{4 \cdot F_{i}}{\pi \cdot d_{f}^{2} \cdot E_{f, i}}$

Where $E_{f, i}$ is the tangent tensile modulus at the start of the stress-strain diagram. Given the variability of diameter and tensile modulus within each fibre population, $\varepsilon_{i}$ is also variable. According to ISO 11566 [37], the strain range for measuring the modulus for carbon fibres with a failure strain higher than $1.2 \%$ should be $0.1-0.6 \%$. Only the HS40 carbon fibre does not have a failure strain in that range; for HS40 the tensile modulus should be measured between $0.1-0.3 \%$. For the fibres with $\varepsilon_{i}$ higher than $0.1 \%$, the initial strain was used as the start of the strain range for modulus measurement.

If the fibre tensile behaviour were linear, one iteration of the compliance calibration method would be sufficient to determine accurately the correction for the machine compliance. The fact that carbon fibres stiffen with the applied strain introduces errors in the determination of the machine compliance. By updating the strain, the data points used to calculate the compliance are not within the respective strain range anymore as the stress-strain diagram is shifted. The machine compliance determination is repeated using the updated strains and new compliances associated with each fibre. The machine compliance is shown in Figure $2 \mathrm{~b}$ as a function of the iteration number for the T700S carbon fibre. The first iteration leads to a deviation (11\% for the T700S fibre, see Figure $2 \mathrm{~b}$ ) of the real machine compliance due to the use of the incorrect strain range in the load-displacement diagram in the first iteration.

After applying the compliance calibration, it is now possible to determine the variation of the fibre stiffness with the applied strain. The elastic modulus was determined for a strain range of $0.3 \%$ and with a step of $0.05 \%$. This means that the first stiffness point would correspond to the strain between $0-0.3 \%$, the second between $0.05-0.35 \%$ and so on. The average variation of stiffness $\Delta E_{f}$ and initial stiffness $E_{f, 0}$ can be determined by linear regression of the stiffness-strain measurements $E(\varepsilon)$ in equation 3 : 
$E(\varepsilon)=E_{f, 0}+\Delta E_{f} \cdot \varepsilon$

The fibre strength for each fibre was determined by extracting the maximum stress carried by the fibre prior to its failure. A two-parameter Weibull distribution (see equation 4) was then determined for each fibre type, using the Matlab 2018a function wblfit, which returns the maximum likelihood estimators for the Weibull modulus, $m$, and scale parameter, $\sigma_{0}$. The same function also returns the $95 \%$ confidence intervals on the Weibull parameters. In equation $4, P$ is the probability that a fibre element with a length $L$ fails when an axial stress $\sigma_{f}$ is applied to it, $L_{0}$ is the reference gauge length, $\sigma_{0}$ is the Weibull scale parameter and $m$ is the Weibull modulus.

$P=1-\exp \left[-\left(\frac{L}{L_{0}}\right) \cdot\left(\frac{\sigma_{f}}{\sigma_{0}}\right)^{m}\right]$

\subsubsection{Composite tensile tests}

Macroscale tensile tests were performed on a Zwick Z100 equipped with a $100 \mathrm{kN}$ load cell, according to ASTM D3039-08 [38]. The gauge length was $150 \mathrm{~mm}$, leaving $50 \mathrm{~mm}$ of the specimen on each side to be gripped by the tensile machine. No end tabs were used as the tests were conducted only to determine the tensile modulus. Sandpaper was used between the specimens and the grips to ensure that no slippage would occur. The displacement rate was $1 \mathrm{~mm} / \mathrm{min}$.

An optical extensometer with an approximate gauge length of $70 \mathrm{~mm}$ was used to measure the strain on the surface of the specimens. The tensile machine had an integrated system to measure the relative displacement between the two reference stickers to measure the surface strains. As the goal of these tests was merely to measure the composite stiffness, the test was interrupted at an applied strain of $0.8 \%$. This conserved the integrity of the specimen and allowed to measure the fibre volume fraction more accurately (see section 2.2.4)

\subsubsection{Fibre volume fraction measurement}

The fibre volume fraction was measured according to method II of ASTM D3171. The gauge section of each specimen was separated from the gripped region to ensure that the volume fraction measured corresponded only to the load carrying portion of the specimen. The dimensions of each specimen were measured with a calliper, ensuring a maximum error of $0.01 \mathrm{~mm}$ in each direction. The three dimensions 
(length, width and thickness) were used to calculate the specimen volume. The mass of each specimen was measured with a high precision scale, ensuring a maximum error of $0.1 \mathrm{mg}$. Using the mass and volume of each specimen, their density, $\rho_{c}$, was determined.

Using the densities of the composite and its constituents, the fibre volume fraction can be calculated. The density of the HS40 and HYBON 2026 fibres, $\rho_{f}$, was considered to be $1.85 \mathrm{~g} / \mathrm{cm}^{3}$ and $2.54 \mathrm{~g} / \mathrm{cm}^{3}$, as mentioned in the respective data sheets. The density of the 736LT matrix, $\rho_{m}$, was measured on neat resin specimens and is $1.20 \mathrm{~g} / \mathrm{cm}^{3}$. For each specimen, the volume fraction, $v_{f}$, was determined using equation 5:

$v_{f}=\frac{\rho_{c}-\rho_{m}}{\rho_{f}-\rho_{m}}$

\subsubsection{Optical microscopy}

A Leica DL ILMC HC inverted light microscope was used to perform optical microscopy on the crosssection of composite specimens. The images were captured with a digital camera Nikon Coolpix 990. The goal was to determine the fibre diameter of the fibres embedded in the matrix. Optical microscopes have a limited resolution of $380 \mathrm{~nm}$, corresponding to the minimum wavelength of visible light. To decrease the human error in measuring the fibre diameter, an ellipse was fitted on the perimeter of the fibre using several points along this perimeter. The ellipse was fitted so that it minimised the distance to each of the manually identified points on the fibre perimeter. The shortest axis of the ellipse corresponds to the fibre diameter as the fibre may be misaligned in the composite. This approach enabled a sub-pixel size resolution.

\section{Results and discussion}

\subsection{Fibre diameter}

The fibre diameter was measured for all the fibres using the laser diffraction system (LDS). Figure 3 shows a histogram of the fibre diameters for each fibre type. Each histogram contains a dashed line that represents the nominal fibre diameter according to the manufacturer's data sheet. The nominal fibre diameter was calculated by dividing the roving linear density by the number of filaments and fibre 
density. The statistical nature of the nominal diameters (mean, median ...) is not specified in the datasheets.

The average diameter value was always lower than the datasheet value. The difference between the two is smaller than $0.5 \mu \mathrm{m}$ for all carbon fibres. It is possible that the diameter is being underestimated by the laser diffraction system but the difference seems to be acceptable. If the measured diameter values were increased to reflect the difference between the average measured diameter and the nominal diameter, the tensile strength would decrease between 140 and $400 \mathrm{MPa}$ depending on the fibre type. This would translate to a decrease of the Weibull scale parameter of the same order of magnitude but the Weibull modulus would retain its value. The other possibility is that the linear density and/or fibre density are also approximated in the manufacturer's data sheet, leading to the described difference. As the laser diffraction system was calibrated for carbon fibres, the tensile modulus and strength are calculated in this paper using the measured fibre diameter values.

The coefficient of variation of the fibre diameter varied between $4.6 \%$ and $6.7 \%$ for the carbon fibres but was higher $(10.3 \%)$ for the E-glass fibres. The coefficient of variation measured for the carbon fibres is in line with other measurements found in the literature [22,29,32,39-42]. The coefficient of variation measured for the E-glass fibre was higher than for other E-glass fibres reported in the literature $[19,30,41,43,44]$. This can be attributed to the specific fibre type.

During the experiments, the LDS failed to measure the diameter of $32 \%$ of the HS40 fibres mounted on the device. Those fibres were not taken into account for the results. No correlation was found between the fibre diameter and fibre strength or stiffness for this fibre type (see Figure 4a), so the results for mechanical properties are believed to be unbiased. Given that the histogram for this fibre does not show a left tail (see Figure 3d), it is likely that the smaller fibre diameters were not measured due to a limitation in the measurement method. To confirm this hypothesis, optical microscopy was performed to observe the cross-section of composite specimens with this fibre type. The results, in Figure 4b, show that the optical microscopy was capable of capturing the same peak in the histogram as in the LDS, but also the left tail of the distribution, absent in the LDS measurements. This tail does not account for all missing $32 \%$ of the population, hencesome fibres with larger diameter were also not measured. 
The HYBON 2026 glass fibres show a much broader distribution of fibre diameters than carbon fibres, in relation with the average value. The average fibre diameter measured by the LDS was $10.3 \%$ lower than the nominal diameter referenced by the manufacturer $(15 \mu \mathrm{m})$. The LDS had problems with the measurement of the glass fibre diameters due to its transparency. Performing the calibration using two glass fibres with previously measured diameter proved to be difficult due to the small difference between the diameter of the fibres used. The calibration was therefore performed using a glass fibre and a metallic wire of higher diameter, which proved to be an inaccurate method. All the fibre diameters measured with the LDS were hence increased by $10.3 \%$ to account for the measurement errors. All the results for the HYBON 2026 fibres shown in this article take into account this correction. This may not be a conventional method, but it brings the average diameter in accordance with the manufacturer's data on fibre diameter.

\subsection{Tensile modulus}

Figure 5 shows the variability of the tensile modulus for each fibre population. The average tensile modulus is quoted in the figure for each fibre type along with the respective $95 \%$ confidence interval. Some fibres presented sudden drops in load in the load-displacement diagrams. These fibres were not considered for the results presented in Figure 5.

All the carbon fibre populations show a tensile modulus in agreement with the manufacturer's data sheet. For the T700S and 34-700, the datasheet value is within the confidence interval of the fibre population. The tensile modulus distribution for the T300 carbon fibre shows two peaks. The same is observed for the diameter distribution for the same fibre. It is known that this fibre type has an elliptical cross-section $[3,45-47]$ and the fibre diameter was measured at only one angular position. The two peaks in the fibre diameter distribution were most likely a result of a measurement of the major or minor axis of the fibre cross-section. The average tensile modulus and standard deviation were presented in Figure 5c between brackets to highlight the errors present in this measurement. This statement is supported by the observed negative correlation between the measured fibre diameter and the tensile modulus (see Figure 6c). Such correlation was not found for any other fibre type. The other fibre types were also investigated for such 
correlation between fibre diameter and tensile modulus but no correlation was found. The results can be found in Figure 6.

The tensile modulus of the HS40 carbon fibre and HYBON 2026 glass fibre was $6 \%$ and 12\%, respectively, higher than the value reported by the manufacturer. The two methodologies used to measure the fibre diameter of the HS40 fibres match reasonably well (see Figure 4), so the stresses and therefore tensile modulus seems to be calculated correctly. For the HYBON 2026 fibre, there were difficulties in the measurement of the fibre diameter due to the transparency of the fibres (see section 3.1). The deviation of the tensile modulus from the data found in the literature [48] may be related to an error in the diameter measurement.

An alternative method was therefore used to determine the tensile modulus of the HS40 and HYBON 2026 fibres. UD composite specimens were produced using these fibres (see section 2.1.2) and tested in tension. This method is similar to the impregnated bundle test, which is commonly used by the fibre manufacturers in setting up their data sheets. Figure 7 shows the stress in the fibres back-calculated from the composite stress-strain diagram. The average fibre stress, $\bar{\sigma}_{f}$, can be back-calculated through equation 6 as a function of the applied strain, $\varepsilon$.

$\bar{\sigma}_{f}(\varepsilon)=\frac{\sigma_{c}(\varepsilon)-\left(1-v_{f}\right) \cdot \sigma_{m}(\varepsilon)}{v_{f}}$

$\sigma_{c}$ is the composite stress measured in the composite tensile tests and $\sigma_{m}$ the stress carried by the matrix. The stress carried by the matrix was interpolated from the 736LT matrix's stress-strain curve. The fibre volume fraction was measured using ASTM D3171 and used to back-calculate the stress in the fibres.

The tensile modulus of the HS40 carbon fibres measured through the composite tests shows high variability. The average value, in the $0.1-0.3 \%$ strain range, was $400.6 \pm 42.7 \mathrm{GPa}$, a value lower than the one reported by the manufacturer (see Table 1). The HS40 fibre is a high modulus fibre and data reported for other high modulus carbon fibres shows that these fibres tend to be very anisotropic [49]. It is therefore possible that a small variation in fibre misalignment between specimens explains the variability in the fibre stiffness. The classical laminate theory was then used to determine the magnitude of the angle that a UD composite needs to be shifted from the loading direction to obtain the differences observed. An 
angle below $3^{\circ}$ is sufficient to explain the difference between $446.5 \mathrm{GPa}$ (measured in single fibre tests) and 400.6 GPa (measured in a composite). Carbon fibre composites were shown in the literature to have a standard deviation of the fibre misalignment of $0.9-1.3^{\circ}[50]$. The fibre manufacturer, although this is not reported in the data sheet, most likely determined the fibre tensile modulus using the impregnated fibre bundle test and had similar misalignment issues. The tensile modulus measured in the single fibre test therefore seems to be the more accurate method for the HS40 carbon fibre.

The glass fibres in a single fibre test have a tensile modulus of $92.4 \pm 0.7 \mathrm{GPa}$, which is higher than the tensile modulus value found in literature [48] for this fibre type (82.7 GPa). The calculation of the tensile modulus was performed using the corrected fibre diameter, as explained in section 3.1. The backcalculated fibre tensile modulus in the composite test was $83.5 \pm 2.5 \mathrm{GPa}$ for the 10 specimens tested. For this set of specimens, the misalignment angle to explain the difference between the value measured in the single fibre testing and the UD composite tensile tests would have to be $5.4^{\circ}$. This is also consistent with values measured in the literature [51] but the difficulties in measuring the fibre diameter may have also influenced the calculation of the tensile modulus in the single fibre tests. If the average diameter were $15.86 \mu \mathrm{m}$ rather than $15 \mu \mathrm{m}$, the fibre tensile modulus would match the value in the literature [48]. It is likely that the difference between the tensile modulus measurements lies in a combination of the two effects: fibre misalignment and diameter measurement inaccuracies.

Carbon fibres have been shown to have a stiffening effect with the applied strain [52]. This effect has not been analysed yet for large populations of fibres. It is interesting to analyse not only the average stiffness increase rate for the fibre types tested but also the variability within the fibre population. Two parameters in the fibre stiffening are analysed here: the rate of stiffness increase and the back-calculated initial stiffness (see section 2.2.2). Figure 8 shows the stiffness-strain diagram for a representative fibre of each fibre type. The average values for $\Delta E_{f}$ and $E_{f, 0}$ are displayed along with their $95 \%$ confidence interval.

All carbon fibre types show increase of stiffness with the applied strain. The results found in the literature [52-57] for carbon fibres show a stiffness increase ranging from $7.3 \%$ [52] to $27.7 \%$ [57] of the initial stiffness per $1 \%$ of increase in strain. It should be noted that the results presented by Curtis et al. [52] are for an older generation of carbon fibres and for only one fibre. For fibres of recent generations, the works 
of Djordjevic et al. [53] and Kant and Penumadu [57] should be referred to, as they present results for the T300 and T700S carbon fibres tested in the present paper. Interestingly, Kant and Penumadu [57] observed that all carbon fibre types they tested had a stiffness increase of $24.4-27.7 \%$ relative to the initial stiffness per $1 \%$ of increase in strain. This consistency was not observed by Djordjevic et al. [53] or in the results obtained in this paper as the different carbon fibres showed different relative stiffness increase.

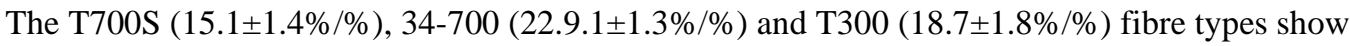
differences in the stiffness-strain rate although the initial stiffness is similar. The HS40 carbon fibre $(14.2 \pm 3.8 \% / \%)$, despite having almost twice the initial stiffness, increases its stiffness at a similar rate as the T700S fibre. Representative stiffness-strain diagrams for each carbon fibre type are shown in Figure $8 a$.

The variation of stiffness with the applied strain in glass fibres has, to the best of the authors' knowledge, not been investigated yet in the literature. Only Huether et al. [58] showed a decrease of the fibre stiffness with the applied load but did not discuss those results. They used a novel method to measure the fibre stiffness using resin beads and digital image correlation. The stiffness decrease in their article may be related to the presence of the beads. The present measurements show that the HYBON 2026 fibre seems to decrease its stiffness with the applied strain (see Figure 8b). There is a justification for carbon fibres to increase their stiffness with the applied strain, as their graphite planes and amorphous phase get better aligned with the loading direction [53]. For glass fibres, however, such preferential orientation is absent. The decreases of stiffness with the applied strain is verified in metals and plastics where it is due to their plastic behaviour. The rate of variation of stiffness was only $-3.0 \pm 1.5 \mathrm{GPa}$ per each $1 \%$ of applied strain, or $-3.7 \%$ of modulus per $1 \%$ of the strain. The cross-sectional area variation due to Poisson contraction of the fibre could be a justification for the measured stiffness decrease. However, with an elastic modulus of $92.4 \pm 0.7 \mathrm{GPa}$ and a Poisson ratio ranging between 0.15 and 0.26 [59], the Poisson contraction only explains a small part of the stiffness decrease: accounting for the Poisson contraction, considering a Poisson's ratio of 0.3 , the stiffness decrease is estimated to be $-2.9 \pm 1.5 \mathrm{GPa}$ per $1 \%$ of applied strain. The force necessary to maintain the molecular bonds of the Si-O structure varies non-linearly with the distance between the atoms [60]. Without breaking any bonds, the energy necessary to deform the fibre 
becomes lower. This could also explain why the deformation promotes the decrease in stiffness of the fibre.

\subsection{Tensile strength}

Figure 9 shows the data points corresponding to the strength of each individual fibre and the line representing the Weibull fit for each carbon fibre type. The Weibull parameters and corresponding 95\% confidence intervals are also shown in the figure.

The T700S, 34-700 and T300 carbon fibres show a deviation from the Weibull distribution for low strength values (see Figure 9a). This was expected and is due to the higher probability of weaker fibres failing during the selection of the fibres from the bundle. A three-parameter Weibull distribution could have been applied to these fibre types based on the interpretation of Islam et al. [14]. However, the low strength threshold implies that only fibres that are weaker than that threshold were not measured. Based on the author's experience, although weaker fibres are more likely to break during the selection, fibres that are more entangled in the bundle were also more likely to fail in the process. Those fibres are not necessarily on the weaker side of the distribution but were still not measured. Setting the threshold is also not a straightforward task, as it may be different for the different fibre types. For the HS40 carbon fibres and HYBON 2026 glass fibres, setting that threshold accurately would not be possible, for example.

The T700S and 34-700 fibres have similar Weibull scale parameter, $\sigma_{0}$, but the Weibull modulus values, $m$, are significantly different. The lower Weibull modulus of the T700S fibres means that these fibres have more variable strength. Also a lower Weibull modulus for fibre strength is shown to lead to higher composite average strength, provided the Weibull scale parameter is the same [33]. This is because the broader strength distribution contains more strong fibres, which make it difficult for fibre break clusters to grow. The disadvantages of high variability may be overcome by advantages of higher average strength for certain design requirements.

The T300 fibres have a lower $\sigma_{0}$ in comparison with $34-700$, which is expected as they have a lower mean tensile strength. Although the HS40 fibres have a lower mean tensile strength compared to the T700S and 34-700 fibres, their $\sigma_{0}$ value is measured to be higher. As mentioned in section 3.2, this may 
be due to (unknown) differences in fibre misalignment for these fibre types, which affect the manufacturer's data. The higher anisotropy of high modulus carbon fibres [49] compared to standard modulus carbon fibres [61] can contribute more to this misalignment issue. The HS40 fibres also have a lower fibre diameter and can suffer a stronger effect of fibre pre-selection during the preparation of the single fibre tests. However, Figure 9a show does not show a deviation from the Weibull estimator for low strength values and therefore the pre-selection does not seem to be an issue for this fibre type.

The glass fibres have a lower stiffness than all the carbon fibres tested. The strength for this fibre type is lower than any of the carbon fibres tested, as can be seen in Figure 9b. The Weibull modulus is higher (the strength variability within the population is lower) for this fibre type than for T300, T700S and 34700 carbon fibres, and is the same (within the statistical confidence) as for HS40. The deviation from the Weibull distribution at low strength values occurred in the opposite direction for the HYBON 2026 fibre than for the carbon fibres. The cross-section of these fibres is larger and so the force to break them is higher. This hinders the fibre pre-selection effect observed for carbon fibres. However, this does not fully explain the deviation in the upwards direction. It was verified though that when half of the results were excluded from the analysis, the deviation occurred in the downwards direction. A different fibre population could therefore yield a different deviation.

The data obtained for these fibre types can be also compared with the data presented in the literature $[13,17-30]$ with the single fibre test as a testing technique (see

Table 2), scaled to the same gauge length $(12 \mathrm{~mm})$ using equation 4 . The results in the literature show a high inconsistency even for the same fibre type. Most authors only test between 20 and 50 fibres in their studies though, which can lead to errors in the estimation of the Weibull parameters. No data was found in the literature for the HS40 carbon fibre.

The Weibull parameters estimated for the T700S and HYBON 2026 fibres seem to be within the range of parameters reported in the literature. The Weibull shape parameter measured for the T700S carbon is close to the lower limit of the reported values. Fortunately, the lowest Weibull shape parameter reported $\left(\sigma_{0}=4.43 \mathrm{GPa}, \mathrm{m}=3.65\right)$, was estimated using the same type of equipment and methodology[14], giving confidence to the results presented here. The 34-700 fibre shows a similar Weibull scale parameter to the 
one reported in the literature but a much lower shape parameter. Only one study was found in the literature for this fibre type [39] and only 20 tests were performed by the authors. The number of tests is insufficient to accurately characterise the Weibull modulus but it may be enough to determine the scale parameter. The results for the T300 carbon fibre show the highest deviation from the literature values. The difficulties in measuring the fibre cross-sectional area for this fibre type may be the origin of the inconsistency between studies. A more detailed study considering the actual cross-sectional area for this fibre type should be performed to confirm the findings in this paper.

\subsection{Size of the sampling for determination of Weibull parameters}

The size of the confidence intervals of the Weibull parameters depends on the number of tests performed for each fibre type. Note that that for the T700S and 34-700 fibres, the confidence intervals for the Weibull modulus lie within $11 \%$ of the average value. The number of tests performed for these fibre types was 217 and 170, respectively. For the T300 and HS40 carbon fibres, the number of tests performed was about 100 (see Table 1) and the confidence interval is above 13\% of the mean for these fibre types. A high number of single fibre tests is necessary to accurately determine the Weibull parameters $[33,62,63]$. To analyse the dependency of the accuracy of identification of the Weibull distribution parameters on the sampling size, a numerical experiment was done. With the high number of tests performed in this study, it is possible to analyse the effect of the sample size with real data.

Using the experimentally obtained fibre strengths for each fibre type, the sampling size, $\mathrm{N}_{\text {sample }}$, was varied between 50 and the number of tests performed for each fibre type. According to the sampling size, $\mathrm{N}_{\text {sample }}$ results were randomly selected from the data set and a Weibull distribution was fitted to the selected results. This process was repeated 20,000 times for each sampling size. An ellipse was then fitted to all the data points in a Weibull scale parameter-Weibull modulus diagram (see Figure 10). Each ellipse represents the $95 \%$ confidence interval for the Weibull parameters obtained for each sampling size.

Figure 10a to $10 \mathrm{e}$ shows the variability of the Weibull parameters for each fibre type depending on the sampling size. The colour of the ellipse is directly related to the sampling size, as indicated by the colour 
bar. The area of the ellipse decreases as the number of experimental results increases. The centre of the ellipse corresponds to the estimated Weibull parameters using the entire data set. Given that the Weibull scale parameter measured using the entire data set is located in the middle of the ellipses obtained with smaller data sets, it is equally likely to over- or underestimate the Weibull scale parameter.

The Weibull parameters can be severely wrongly estimated if a small number of tests is performed. The Weibull modulus for the T700S carbon fibre, for example, can vary between 3.1 and 5.1 if only 50 tests are performed. This represents a deviation of \pm 1 from the value calculated with the entire data set. A deviation of such magnitude in the estimation of the Weibull modulus can change the prediction of the strength variability of the composite bundle [64-67]. This shows the importance of performing a high number of tests when the results are applied to modelling of composite strength. Most authors have tested between 20-50 fibres and this is not enough for an accurate estimation of the Weibull parameters. The aspect ratio of the ellipse is higher, making the estimation of the Weibull modulus more difficult than the Weibull scale parameter. Using longer fibres could be a way to address this issue, as the strength of a fibre of $24 \mathrm{~mm}$ is approximately equivalent to the minimum strength of two fibres of $12 \mathrm{~mm}$ each. It would be interesting to perform this analysis with fibres of different sizes to understand if the sampling size can be smaller to achieve the same level of accuracy.

The angle of the ellipse's major axis with the horizontal is also an interesting feature to analyse. This angle is about $90^{\circ}$ for the T700S, 34-700 and T300 carbon fibres. For the HS40 and HYBON 2026, however, the angle is smaller. If an artificial set of fibre strengths is generated using equation 4 , the angle of the major axis with the horizontal is always lower than $90^{\circ}$. The deviations from the Weibull distribution in the T700S, 34-700 and T300 carbon fibre due to fibre pre-selection may be the source of the orientation of the ellipses. The HS40 and HYBON 2026 show less evidences of fibre pre-selection and the angle of the major axis of the ellipses is also smaller than $90^{\circ}$. Using this analysis, the data sets that result in ellipses with angles of $90^{\circ}$ may show signs of the fibre-preselection, as is the case of the T700S, 34-700 and T300 fibres.

The size and shape of the ellipses when a sampling size of 50 is considered can be compared in Figure 10f. It is noticeable that the T700S fibre, with a lower Weibull modulus produces a larger uncertainty and 
therefore its corresponding ellipse is larger. Although with a difference in Weibull modulus of 0.5 between the 34-700 and T300 fibres, their relative uncertainty in the parameter estimation is similar. Finally, the HS40 and HYBON 2026, due to the reasons presented above, show an angle in the orientation of the ellipse. Still, the HS40 fibre ellipse's size is the smallest, showing a higher certainty in the estimation of the parameters even when the sampling size is small.

\section{Conclusions}

This paper provides large data sets of fibre properties that can be used by the composite materials community. Strength models of unidirectional fibre bundles and micromechanical models of composites in general can use the data provided by this paper to predict the composite properties. The fibre diameter, tensile modulus and strength is also required to experimentally determine other fibre properties such as the fibre-matrix interface strength and fracture toughness, among others. Although the fibre manufacturers provide the average properties, their variability, essential for accurate modelling, can only be obtained through the testing of each fibre individually.

Reliably describing the fibre strength Weibull distribution using single fibre tests is not straightforward, and several conclusions can be drawn from the present work:

- Sampling size: Large datasets are required to properly estimate the Weibull parameters. The sets of $~ 50$ fibres, often used in literature, may lead to a deviation of the Weibull modulus of \pm 1 from the value estimated using $\sim 200$ data points. Testing longer fibres could be a possibility to reduce the number of fibres tested but it remains unverified how the fibre length can reduce the uncertainty for lower sample sizes.

- Fibre diameter: Measuring the fibre diameter efficiently on such large data sets was achieved using a laser diffraction system. This system only works with opaque fibres though, hindering its use on glass fibres. Optical or vibrational methods would be more appropriate to measure the diameter of these fibres. The non-circularity of some fibres such as the T300 carbon fibre also implies that the "fibre diameter" should be measured for several angular positions, when an LDS system is used. 
- Compliance calibration: The compliance calibration on fibres with a non-linear stress-strain curve cannot be done in one step. The estimated displacement range should be adjusted in each iteration. This applies to the level of non-linearity characteristic for carbon fibres.

We have confirmed the previous data on stiffening of carbon fibres, and have found unexpected behaviour for the glass fibre: the HYBON 2026 fibre slowly decreases the stiffness with the applied strain, with the rate of $\sim 4 \mathrm{GPa}$ per $1 \%$ of the strain. While certain sources of this behaviour can be suggested, the investigation of the phenomenon is a subject of future work.

\section{Acknowledgments}

The research leading to these results has been done within the framework of the FiBreMoD project and has received funding from the European Union's Horizon 2020 research and innovation programme under the Marie Skłodowska-Curie grant agreement No 722626. SVL holds the Toray Chair for Composite Materials, the support of which is acknowledged. The stress-strain diagram of the 736LT matrix was experimentally obtained by Christian Breite, whom the authors thank for his efforts.

\section{References}

[1] A.A. Griffith, The Phenomenon of Rupture and Flow in Solids, Philos. Trans. R. Soc. London. 221 (1921) 163-198.

[2] F. Tanaka, T. Okabe, H. Okuda, I.A. Kinloch, R.J. Young, Factors controlling the strength of carbon fibres in tension, Compos. Part A Appl. Sci. Manuf. 57 (2014) 88-94.

[3] K. Naito, Y. Tanaka, J.M. Yang, Y. Kagawa, Tensile properties of ultrahigh strength PAN-based, ultrahigh modulus pitch-based and high ductility pitch-based carbon fibers, Carbon N. Y. 46 (2008) 189-195.

[4] M.R. Gurvich, A.T. DiBenedetto, S. V. Ranade, A New Statistical Distribution for Characterizing the Random Strength of Brittle Materials, J. Mater. Sci. 32 (1997) 2559-2564.

[5] J. Andersons, R. Joffe, M. Hojo, S. Ochiai, Glass fibre strength distribution determined by common experimental methods, Compos. Sci. Technol. 62 (2002) 131-145.

[6] J. Watanabe, F. Tanaka, H. Okuda, T. Okabe, Tensile strength distribution of carbon fibers at short gauge lengths, Adv. Compos. Mater. 23 (2014) 535-550.

[7] A.S. Watson, R.L. Smith, An examination of statistical theories for fibrous materials in the light of experimental data, J. Mater. Sci. 20 (1985) 3260-3270.

[8] I.J. Beyerlein, S.L. Phoenix, Statistics for the strength and size effects of microcomposites with four carbon fibers in epoxy resin, Compos. Sci. Technol. 56 (1996) 75-92. 
[9] S.L. Phoenix, P. Schwartz, H.H. Robinson, Statistics for the strength and lifetime in creep-rupture of model carbon/epoxy composites, Compos. Sci. Technol. 32 (1988) 81-120.

[10] H. Otani, S.L. Phoenix, P. Petrina, Matrix effects on lifetime statistics for carbon fibre-epoxy microcomposites in creep rupture, J. Mater. Sci. 26 (1991) 1955-1970.

[11] R. Gulino, S.L. Phoenix, Weibull strength statistics for graphite fibres measured from the break progression in a model graphite/glass/epoxy microcomposite, J. Mater. Sci. 26 (1991) 3107 3118.

[12] T. Okabe, N. Takeda, Size effect on tensile strength of unidirectional CFRP compositesexperiment and simulation, Compos. Sci. Technol. 62 (2002) 2053-2064.

[13] H.D. Wagner, S.L. Phoenix, P. Schwartz, A Study of Statistical Variability in the Strength of Single Aramid Filaments, J. Compos. Mater. 18 (1984) 312-338.

[14] F. Islam, S. Bucknell, Y. Leray, A. Bunsell, W. Distribution, C. Interval, Towards accurate and efficient single fibre characterization to better assess failure strength, Proc. 18th Eur. Conf. Compos. Mater. (2018) 24-28.

[15] Y. Swolfs, I. Verpoest, L. Gorbatikh, A review of input data and modelling assumptions in longitudinal strength models for unidirectional fibre-reinforced composites, Compos. Struct. 150 (2016) 153-172.

[16] T. Okabe, N. Takeda, Y. Kamoshida, M. Shimizu, W.A. Curtin, A 3D shear-lag model considering micro-damage and statistical strength prediction of unidirectional fiber-reinforced composites, Compos. Sci. Technol. 61 (2001) 1773-1787.

[17] Z. Chi, T.-W. Chou, G. Shen, Determination of single fibre strength distribution from fibre bundle testings, J. Mater. Sci. 19 (1984) 3319-3324.

[18] T.A. Parthasarathy, Extraction of Weibull parameters of fiber strength from means and standard deviations of failure loads and fiber diameters, J. Am. Ceram. Soc. 84 (2001) 588-592.

[19] M. Herráez, A. Fernández, C.S. Lopes, C. González, Strength and toughness of structural fibres for composite material reinforcement, Philos. Trans. R. Soc. A Math. Phys. Eng. Sci. 374 (2016).

[20] K. Naito, J.M. Yang, Y. Kagawa, The effect of interlayer modifications on tensile properties of high strength PAN-based and high modulus pitch-based hybrid carbon fiber reinforced epoxy matrix composite, in: ECCM 2012 - Compos. Venice, Proc. 15th Eur. Conf. Compos. Mater., 2012.

[21] S. Pimenta, S.T. Pinho, The influence of micromechanical properties and reinforcement architecture on the mechanical response of recycled composites, Compos. Part A Appl. Sci. Manuf. 56 (2014) 213-225.

[22] R. Harikrishnan, P.M.M.C.S. Upadhyay, P.M. Mohite, C.S. Upadhyay, Generalized Weibull model-based statistical tensile strength of carbon fibres, Arch. Appl. Mech. 88 (2018) 1617-1636.

[23] D.T. Burn, L.T. Harper, M. Johnson, N.A. Warrior, U. Nagel, L. Yang, J. Thomason, The usability of recycled carbon fibres in short fibre thermoplastics: interfacial properties, J. Mater. Sci. 51 (2016) 7699-7715.

[24] T. Wu, W. Zhang, X. Jin, X. Liang, G. Sui, X. Yang, Efficient reclamation of carbon fibers from epoxy composite waste through catalytic pyrolysis in molten $\mathrm{ZnCl} 2$, RSC Adv. 9 (2019) 377388. 
[25] W. Liu, S. Zhang, B. Li, F. Yang, W. Jiao, L. Hao, R. Wang, Improvement in Interfacial Shear Strength and Fracture Toughness for Carbon Fiber Reinforced Epoxy Composite by Fiber Sizing, Polym. Compos. 35 (2014) 482-488.

[26] M.H. Berger, D. Jeulin, Statistical analysis of the failure stresses of ceramic fibres: Dependence of the Weibull parameters on the gauge length, diameter variation and fluctuation of defect density, J. Mater. Sci. 38 (2003) 2913-2923.

[27] H.L.H. Yip, S.J. Pickering, C.D. Rudd, Characterisation of carbon fibres recycled from scrap composites using fluidised bed process, Plast. Rubber Compos. 31 (2002) 278-282.

[28] L. Claudio Pardini, L. Guilherme Borzani Manhani, Influence of the testing gage length on the strength, young's modulus and weibull modulus of carbon fibres and glass fibres, Mater. Res. 5 (2002) 411-420.

[29] M. Wada, K. Kawai, T. Suzuki, H. Hira, S. Kitaoka, Effect of superheated steam treatment of carbon fiber on interfacial adhesion to epoxy resin, Compos. Part A Appl. Sci. Manuf. 85 (2016) $156-162$.

[30] R.S. Ginder, S. Ozcan, Recycling of Commercial E-glass Reinforced Thermoset Composites via Two Temperature Step Pyrolysis to Improve Recovered Fiber Tensile Strength and Failure Strain, Recycling. 4 (2019) 24.

[31] P. Zinck, E. Mader, J.F. Gerard, L. Mat, Role of silane coupling agent and polymeric film former for tailoring glass fiber sizings, J. Mater. Sci. 36 (2001) 5245-5252.

[32] S. Bai, K.K.C. Ho, G. Knox, A. Bismarck, Improving the adhesion between carbon fibres and an elastomer matrix using an acrylonitrile containing atmospheric plasma treatment, Compos. Interfaces. 20 (2013) 761-782.

[33] Y. Swolfs, I. Verpoest, L. Gorbatikh, Issues in strength models for unidirectional fibre-reinforced composites related to Weibull distributions, fibre packings and boundary effects, Compos. Sci. Technol. 114 (2015) 42-49.

[34] Dia-stron Ltd, Automated LEX / LDS System LDS0200 Module Manual / Automated Sample Loading System, Www.Diastron.Com/App/Uploads/2018/06/Dia-Stron-LEX-LDS-BrochureV2.Pdf. 1 (2018).

[35] Textechno, FAVIMAT+ (AI)ROBOT2 FAVIGRAPH: Automatic single-fibre-testes, (2015) 112.

[36] American Society for Testing and Materials, ASTM C1557-14 - Standard Test Method for Tensile Strength and Young's Modulus of Fibers, Astm C1557-14. (2014) 1-10.

[37] British Standard Institution, Carbon fibre - Determination of the tensile properties of singlefilament specimens, BS ISO 11566. (1996) 1-5.

[38] American Society for Testing and Materials, Astm D3039/D3039M-08 Tensile Properties of Polymer Matrix Composite Materials, Annu. B. ASTM Stand. (2008) 1-13.

[39] H.L.H. Yip, S.J. Pickering, C.D. Rudd, Characterisation of carbon fibres recycled from scrap composites using fluidised bed process, Plast. Rubber Compos. 31 (2003) 278-282.

[40] K. Naito, J.M. Yang, Y. Tanaka, Y. Kagawa, The effect of gauge length on tensile strength and Weibull modulus of polyacrylonitrile (PAN)- and pitch-based carbon fibers, J. Mater. Sci. 47 (2012) 632-642. 
[41] F.A. Ramirez, L.A. Carlsson, B.A. Acha, Evaluation of water degradation of vinylester and epoxy matrix composites by single fiber and composite tests, J. Mater. Sci. 43 (2008) 5230-5242.

[42] T. Langston, The effects of nitric acid and silane surface treatments on carbon fibres and carbon/vinyl ester composites beforea and after seawater exposure, 2008.

[43] S. Kling, T. Czigany, A comparative analysis of hollow and solid glass fibers, Text. Res. J. 83 (2013) 1764-1772.

[44] L. Yang, J.L. Thomason, Effect of silane coupling agent on mechanical performance of glass fibre, J. Mater. Sci. 48 (2013) 1947-1954.

[45] X. Ma, S. Chen, M. Mei, Y. Li, G. Li, H. Hu, X. He, X. Qu, Microstructure and mechanical behaviors of $\mathrm{T} 700$ carbon fiber reinforced $\mathrm{C} / \mathrm{SiC}$ composites via precursor infiltration and pyrolysis, Mater. Sci. Eng. A. 666 (2016) 238-244.

[46] B.A. Newcomb, H.G. Chae, The properties of carbon fibers, in: Handb. Prop. Text. Tech. Fibres, 2018: pp. 841-871.

[47] D.H. Kim, B.H. Kim, K.S. Yang, Y.H. Bang, S.R. Kim, H.K. Im, Analysis of the microstructure and oxidation behavior of some commercial carbon fibers, J. Korean Chem. Soc. 55 (2011) 819823.

[48] R.T.D. Prabhakaran, T.L. Andersen, C.M. Markussen, B. Madsen, H. Lilholt, Tensile and compression properties of hybrid composites - a comparative study, Proc. 19th Int. Conf. Compos. Mater. (2013) 1029-1035.

[49] N. Baral, H. Guezenoc, P. Davies, C. Baley, High modulus carbon fibre composites: Correlation between transverse tensile and mode I interlaminar fracture properties, Mater. Lett. 62 (2008) 1096-1099.

[50] K.K. Kratmann, L.T. Lilleheden, R. Pyrz, Novel Image Analysis Procedure for Measuring Fibre, Compos. Sci. Technol. 69 (2009) 228-238.

[51] R. Thirumalai, D. Prabhakaran, H. Lilholt, F. Aviles, T. Løgstrup Andersen, H. Knudsen, Fibre waviness and misalignment measurement of unidirectional glass / LPET commingled composites - Effect on mechanical properties, Risoe Int. Symp. Mater. Sci. Proceedings,. 34 (2013) 349-363.

[52] G.J. Curtis, J.M. Milne, W.N. Reynolds, Non-hookean behaviour of strong carbon fibres, Nature. 220 (1968) 1024.

[53] I.M. Djordjević, D.R. Sekulić, M.M. Stevanović, Non-linear elastic behaviour of carbon fibres of different structural and mechanical characteristic, J. Serbian Chem. Soc. 72 (2007) 513-521.

[54] C.P. Beetz, Strain-induced stiffening of carbon fibres, Fibre Sci. Technol. 16 (1982) 219-229.

[55] C.P. Beetz, G.W. Budd, Strain modulation measurements of stiffening effects in carbon fibers, Rev. Sci. Instrum. 54 (1983) 1222-1226.

[56] J.D.H. Hughes, Strength and modulus of current carbon fibres, Carbon N. Y. 24 (1986) 551-556.

[57] M. Kant, D. Penumadu, Dynamic mechanical characterization for nonlinear behavior of single carbon fibers, Compos. Part A Appl. Sci. Manuf. 66 (2014) 201-208.

[58] J. Huether, P. Rupp, I. Kohlschreiber, K.A. Weidenmann, An enhanced method to determine the Young's modulus of technical single fibres by means of high resolution digital image correlation, 
Meas. Sci. Technol. 29 (2018).

[59] J. Hutchins, R. Harrington, Glass, in: Encycl. Chem. Technol., 1966: pp. 533-604.

[60] J. Onoda, M. Ondráček, P. Jelínek, Y. Sugimoto, Electronegativity determination of individual surface atoms by atomic force microscopy, Nat. Commun. 8 (2017) 1-6.

[61] K. Searles, G. Odegard, M. Kumosa, Micro- and mesomechanics of 8-harness satin woven fabric composites: I - Evaluation of elastic behavior, Compos. - Part A Appl. Sci. Manuf. 32 (2001) 1627-1655.

[62] R. Bermejo, P. Supancic, R. Danzer, Influence of measurement uncertainties on the determination of the Weibull distribution, J. Eur. Ceram. Soc. 32 (2012) 251-255.

[63] J.L. Thomason, On the application of Weibull analysis to experimentally determined single fibre strength distributions, Compos. Sci. Technol. 77 (2013) 74-80.

[64] D.G. Harlow, S.L. Phoenix, The Chain-of-Bundles Probability Model for the Strength of Fibrous Materials II: A Numerical Study of Convergence, J. Compos. Mater. 12 (1978) 314-334.

[65] D.G. Harlow, S.L. Phoenix, Probability distributions for the strength of composite materials II: A convergent sequence of tight bounds, Int. J. Fract. 17 (1981) 601-630.

[66] S. Mahesh, S.L. Phoenix, I.J. Beyerlein, Strength distributions and size effects for 2D and 3D composites with Weibull fibers in an elastic matrix, Int. J. Fract. 115 (2002) 41-85.

[67] S.L. Phoenix, I.J. Beyerlein, Statistical Strength Theory for Fibrous Composite Materials, Compr. Compos. Mater. (2000) 559-639.

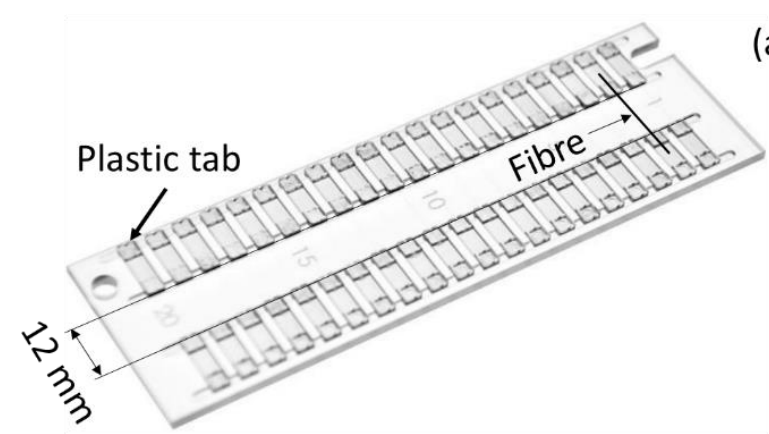

(a)
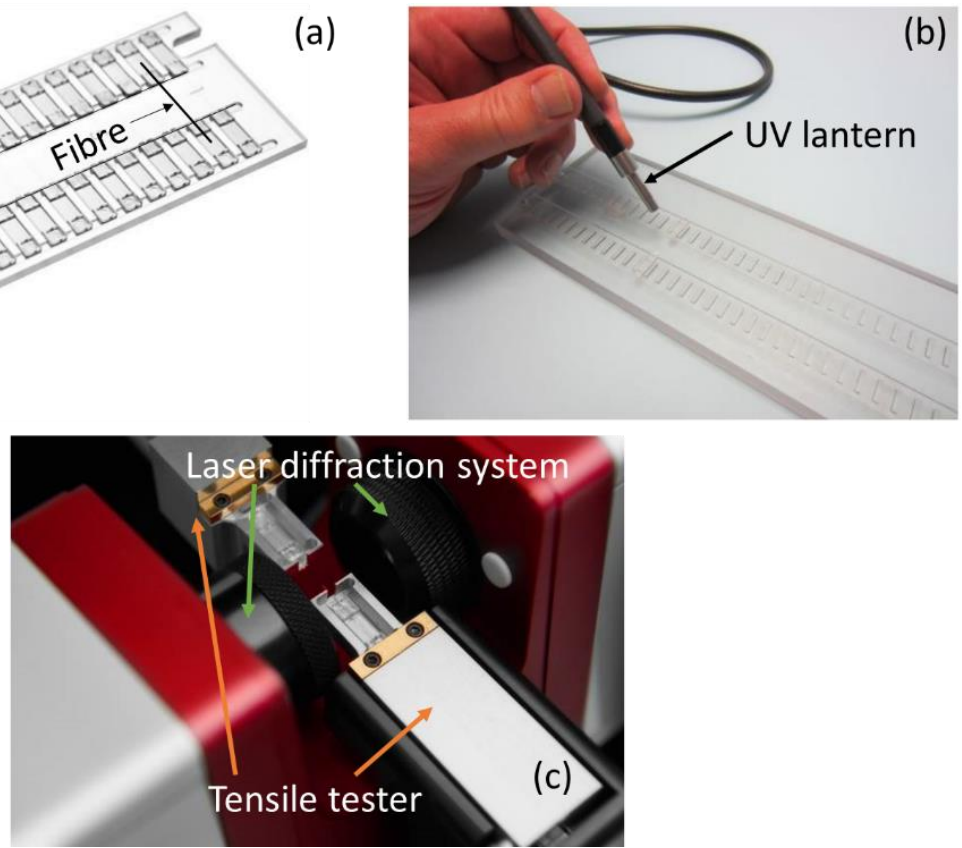

Figure 1: Sample preparation and testing comprising (a) a 20-slot cassette with plastic tabs where fibres are mounted, (b) the UV lantern to cure the adhesive and (c) the automated tensile testing equipment with an incorporated laser diffraction system. 

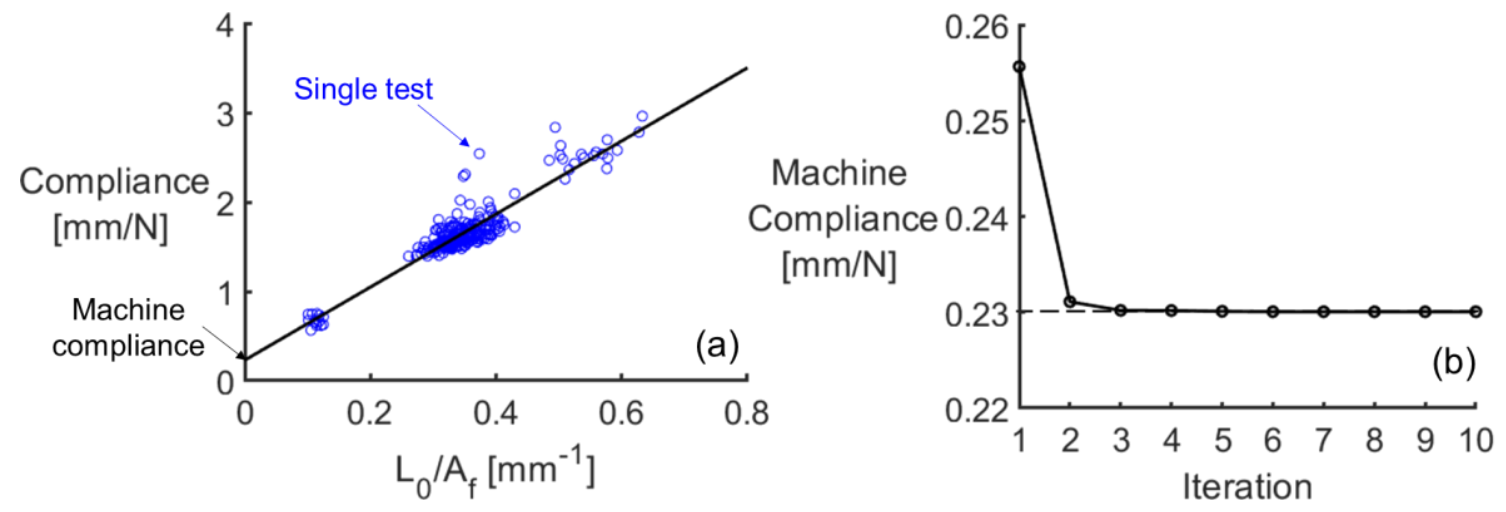

Figure 2: Compliance of the (a) system as a function of the ratio between the gauge length and the fibre cross-sectional area and (b) machine as a function of the iteration number for T700S carbon fibres. The system compliance corresponds to the machine compliance when the gauge length is zero. 

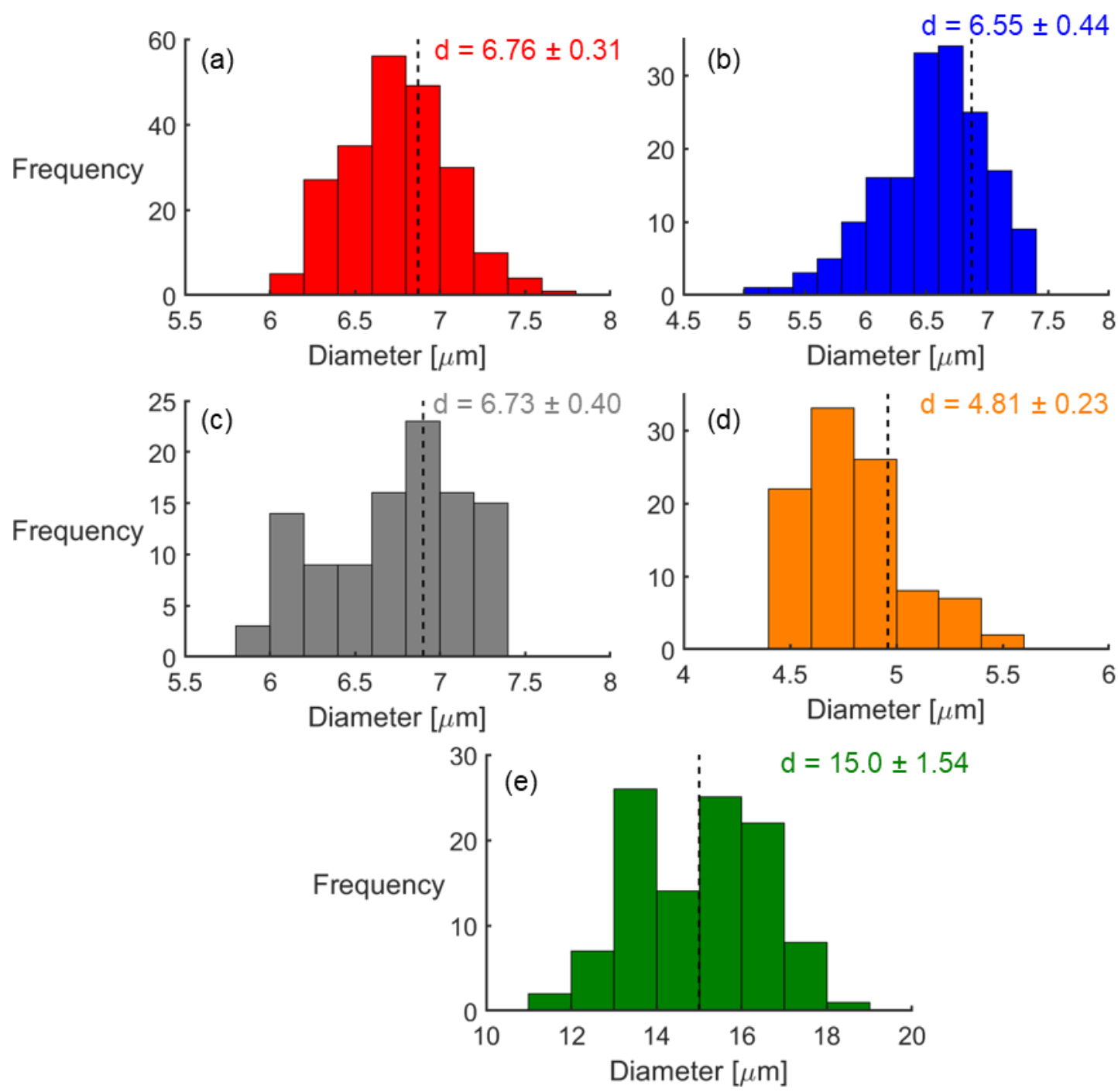

Figure 3: Distribution of the fibre diameter for the fibre populations: (a) T700S, (b) 34-700, (c) T300, (d) HS40 and (e) HYBON 2026 based on LDS measurements. The dashed line represent the data sheet value.
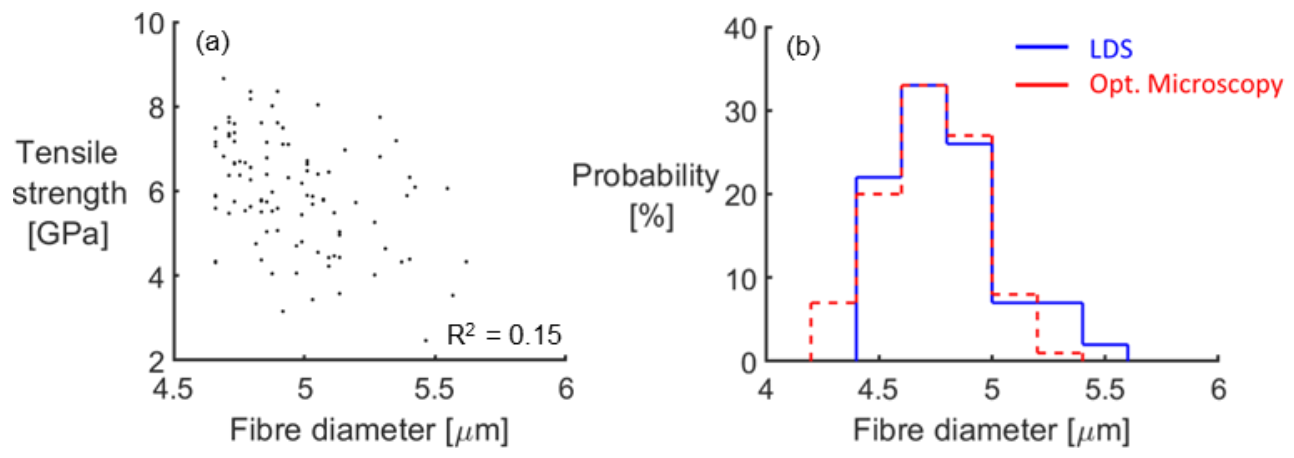

Figure 4: Analysis of the HS40 fibre diameter showing (a) difference between measurements with the LDS and optical microscopy and (b) the correlation between fibre diameter and the fibre's tensile strength. The optical microscopy results show the absent left tail in the LDS histogram. 

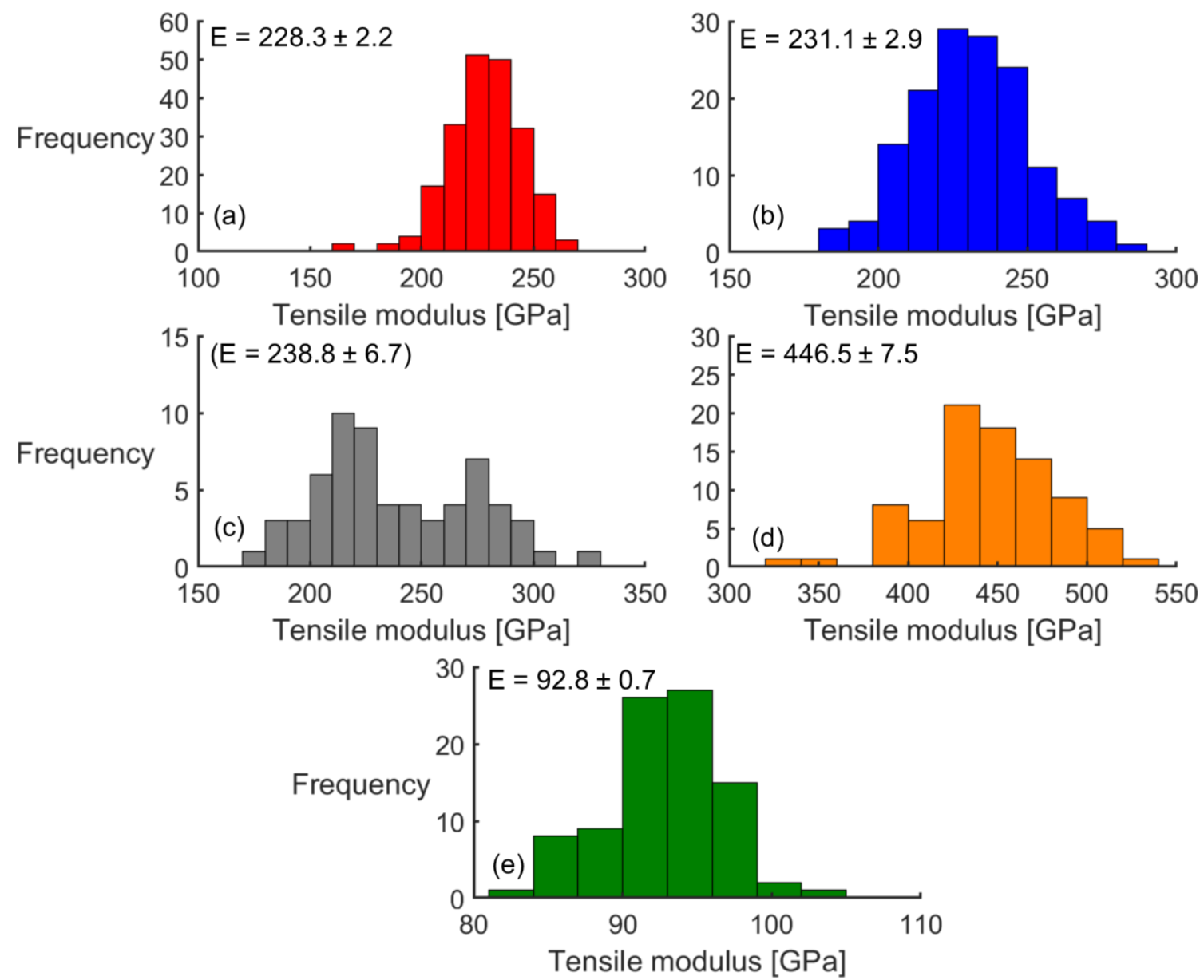

Figure 5: Histogram of the fibre tensile modulus for the (a) T700S, (b) 34-700, (c) T300, (d) HS40 and (e) HYBON 2026 fibres. The presented scatter is the $95 \%$ confidence interval. The tensile modulus presented in (c) should be interpreted carefully. The tensile modulus was measured between $0.1-0.6 \%$ strain for all the fibre types except the HS40 fibre, for which the strain range $0.1-0.3 \%$ was used. 

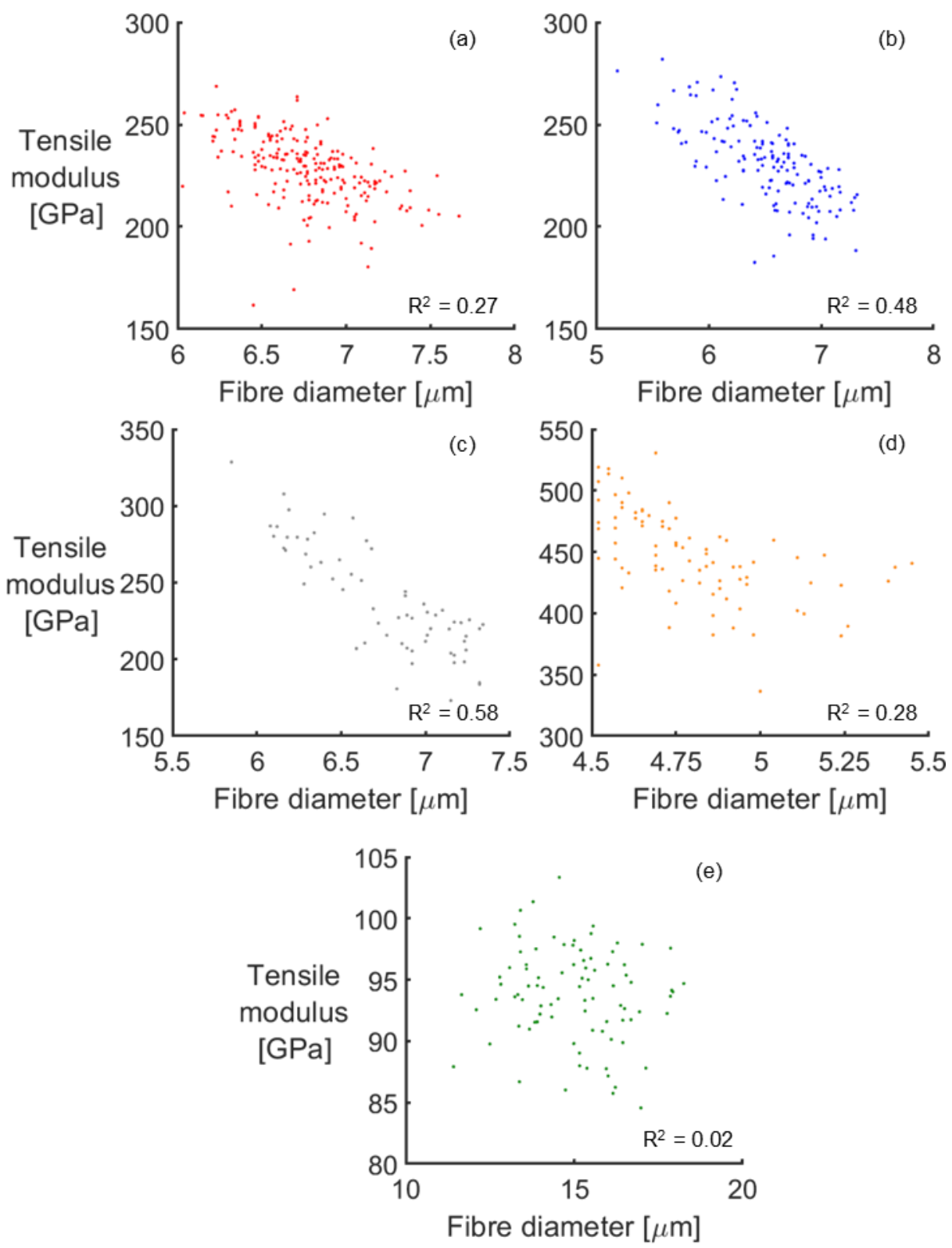

Figure 6: Correlation between the fibre diameter and tensile modulus for the (a) T700S, (b) 34-700, (c) T300, (d) HS40 and (e) HYBON 2026 fibre populations. The tensile modulus was measured between $0.1-$ $0.6 \%$ strain for all the fibre types except the HS40 fibre, for which the strain range $0.1-0.3 \%$ was used. 

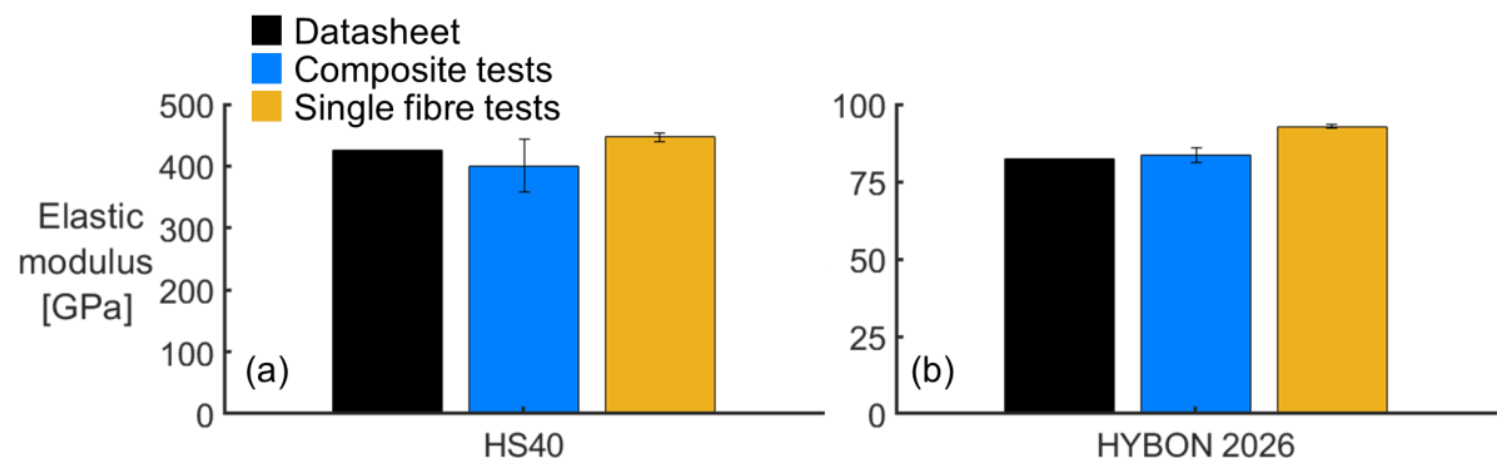

Figure 7: Comparison between the stiffness given by the manufacturer's datasheet and measured using the UD composite tests and single fibre tests for the (a) HS40 and (b) HYBON 2026 fibres. 

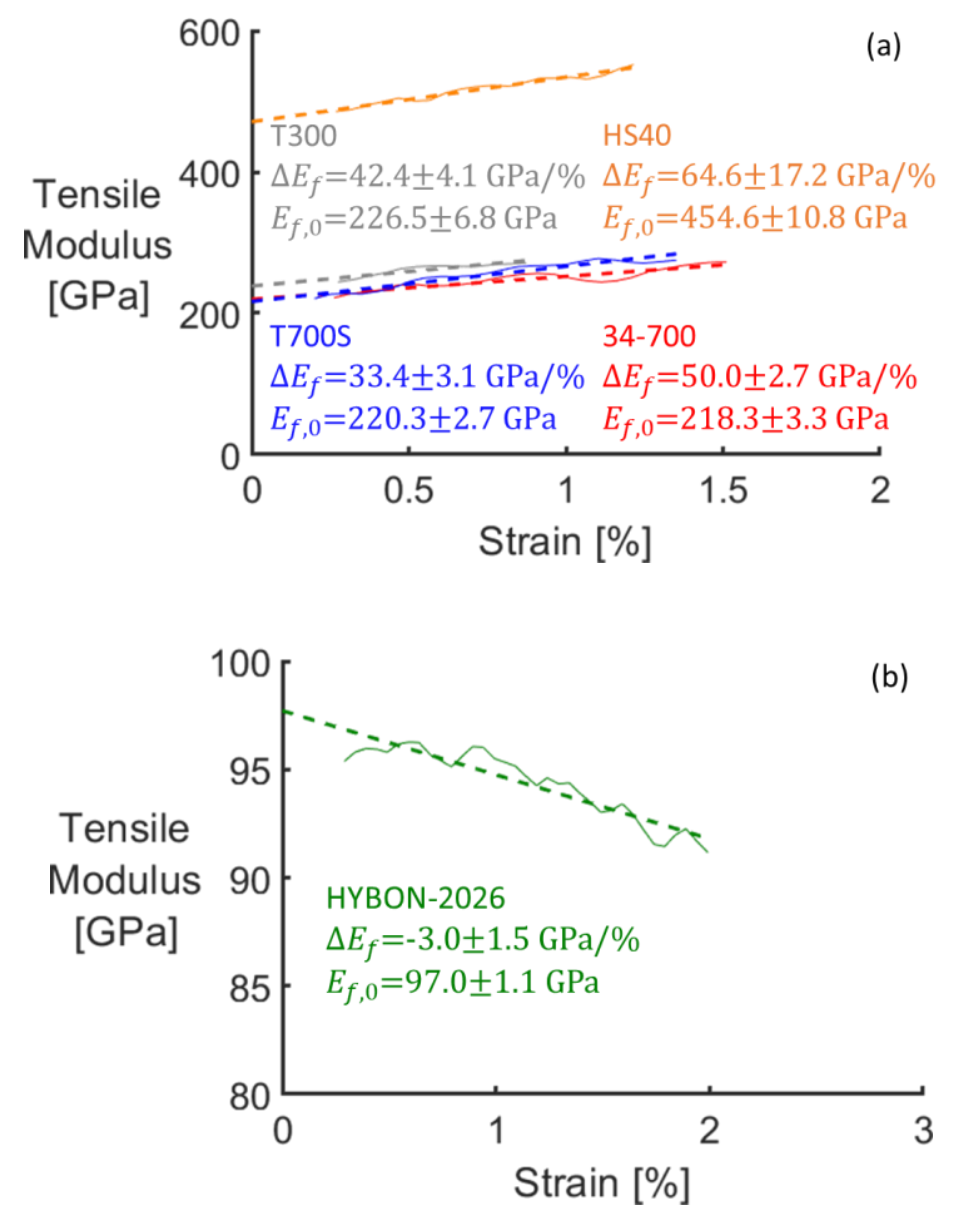

Figure 8: Stiffness-strain diagram for a representative fibre of each (a) carbon fibre and (b) glass fibre type. $\Delta \boldsymbol{E}_{\boldsymbol{f}}$ is the rate of stiffness increase with strain and $\boldsymbol{E}_{\boldsymbol{f}, \mathbf{0}}$ is the initial stiffness of the fibre.
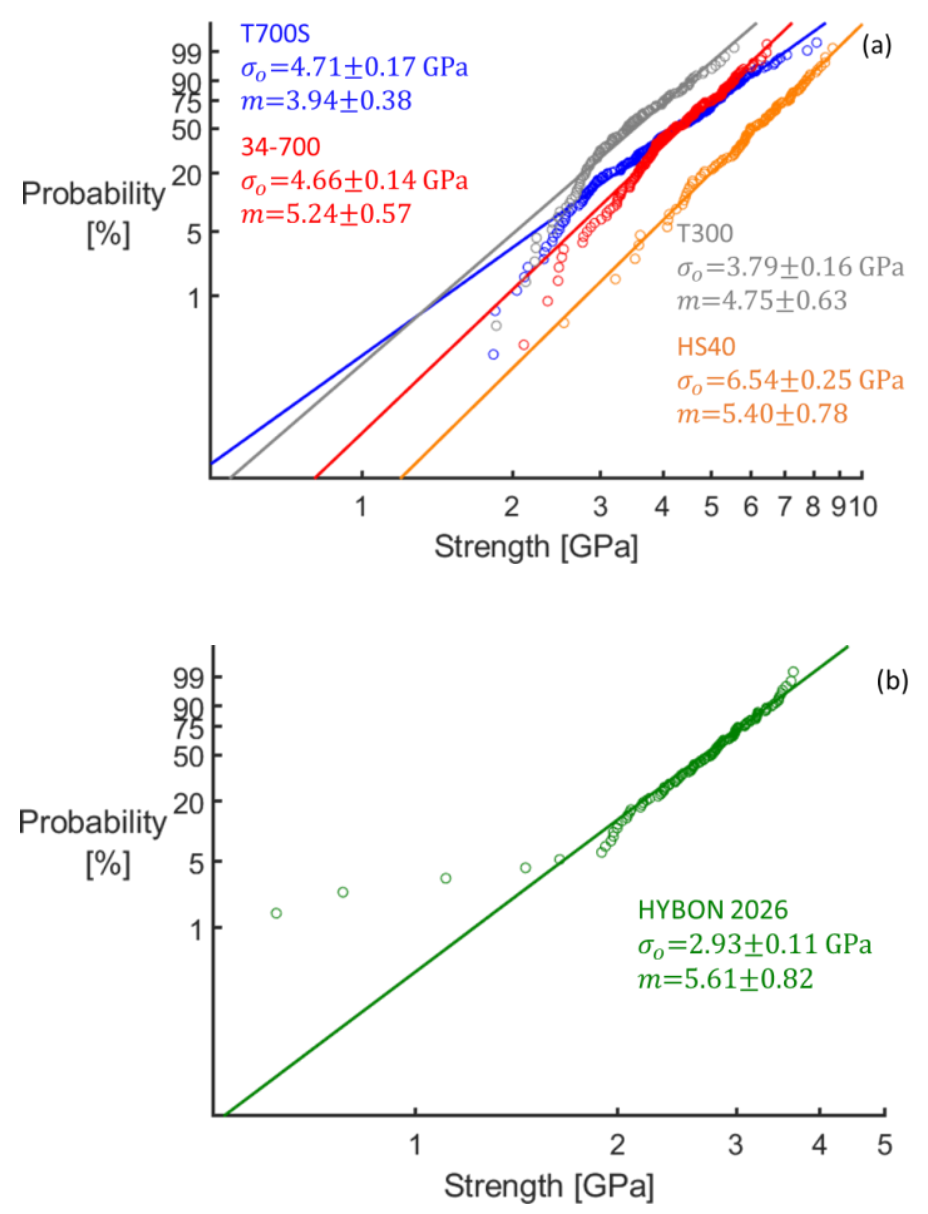

Figure 9: Weibull plots for the tensile strength of the (a) T700S, 34-700, T300 and HS40 carbon fibres and (b) HYBON 2026 glass fibres for a gauge length of $12.24 \pm 0.1$ The deviation corresponds to the $95 \%$ confidence intervals. 

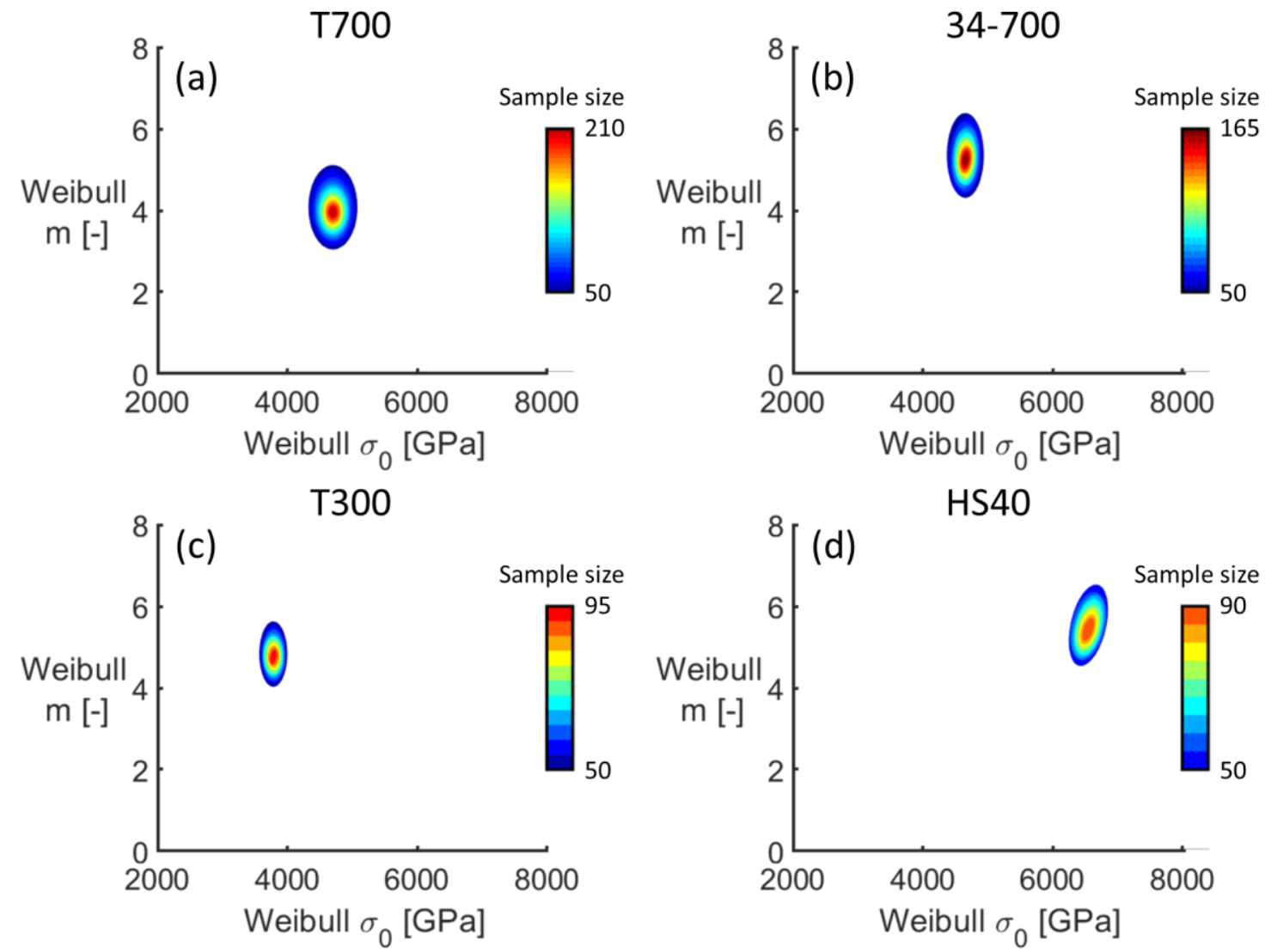

HS40
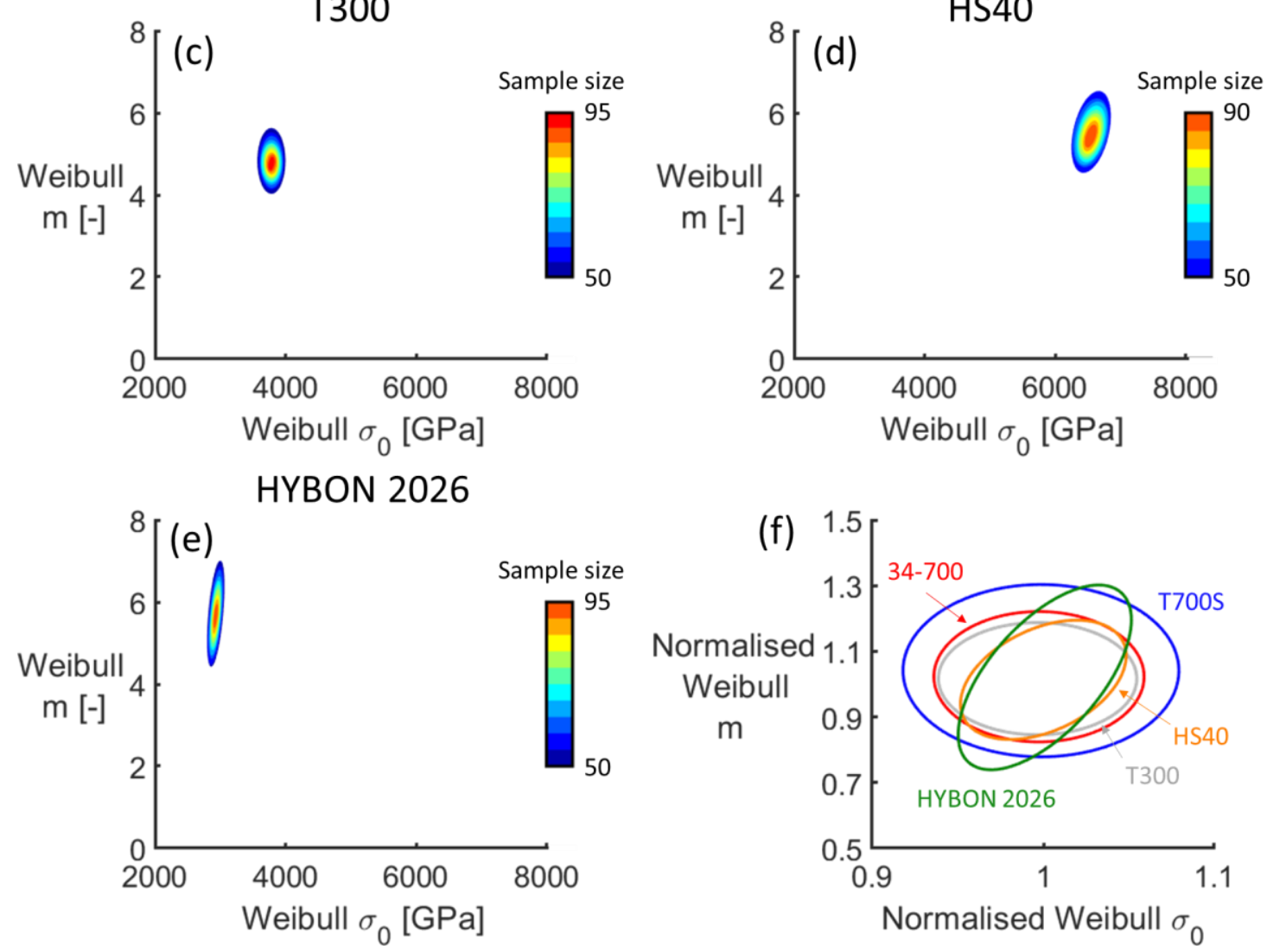

Figure 10: Variability of the Weibull parameters with the sample size. Each plot (a) to (e) corresponds to one of the fibre types tested and plot (f) shows a normalised ellipses for all the fibre types when 50 samples are considered. As the number of tests performed increases, the area of the ellipses decreases. 
Table 1: Nominal properties of the fibres found in the respective data sheets and number of tests performed.

\begin{tabular}{cccccc} 
Fibre type & $\begin{array}{c}\text { Diameter } \\
{[\boldsymbol{\mu m}]}\end{array}$ & $\begin{array}{c}\text { Tensile } \\
\text { modulus [GPa] }\end{array}$ & $\begin{array}{c}\text { Tensile } \\
\text { strength [GPa] }\end{array}$ & $\begin{array}{c}\text { Failure } \\
\text { strain [\%] }\end{array}$ & $\begin{array}{c}\text { Number } \\
\text { of tests }\end{array}$ \\
\hline T700SC-12K-50C & 6.87 & 230 & 4.90 & 2.1 & 217 \\
34-700WD-24K-1.4\%A & 6.87 & 234 & 4.83 & 2.1 & 170 \\
T300B-3K-40B & 6.9 & 230 & 3.53 & 1.5 & 105 \\
HS40-12PDD-AA & 4.96 & 425 & 4.61 & 1.1 & 99 \\
HYBON 2026 (PPG) & 15 & 82 & 2.79 & 3.4 & 105 \\
\hline
\end{tabular}

Table 2: Comparison of the obtained Weibull parameters with the ones found in the literature for the same fibre types (recalculated to the gauge length of $12 \mathrm{~mm}$ ). The HYBON 2026 glass fibre is compared to other E-glass fibres.

\begin{tabular}{ccccc} 
& \multicolumn{2}{c}{ Scale parameter [GPa] } & \multicolumn{2}{c}{ Weibull modulus [-] } \\
Fibre type & This study & $\begin{array}{c}\text { Literature } \\
{[6,12,18-32]}\end{array}$ & & $\begin{array}{c}\text { This study } \\
\text { Literature } \\
{[6,12,18-} \\
\text { T700S }\end{array}$ \\
34-700 & 4.71 & $3.8-6.36$ & 3.94 & $3.65-5.78$ \\
T300 & 4.66 & 4.20 & 5.24 & 7.2 \\
HS40 & 3.79 & $2.84-3.65$ & 4.75 & $5.59-9.35$ \\
HYBON 2026 & 6.54 & - & 5.4 & - \\
\hline
\end{tabular}

